\title{
Relative levels of let-7a, miR-17, miR-27b, miR-125a, miR-125b and miR-206 as potential molecular markers to evaluate grade, receptor status and molecular type in breast cancer
}

\author{
TOMASZ P. LEHMANN ${ }^{1}$, KONSTANTY KORSKI $^{2}$, ROBERT GRYCZKA $^{3}$, MATHEW IBBS $^{2}$, \\ ANNA THIELEMAN $^{4}$, SYLWIA GRODECKA-GAZDECKA $^{3}$ and PAWEŁ P. JAGODZIŃSKI ${ }^{1}$ \\ ${ }^{1}$ Department of Biochemistry and Molecular Biology, University of Medical Sciences, Poznań 60-781; \\ ${ }^{2}$ Department of Pathology, Wielkopolska Cancer Center, Poznań 61-866; \\ ${ }^{3}$ Department of Surgery, Chair and Clinic of Oncology; \\ ${ }^{4}$ Department of Laboratory Diagnostics, University of Medical Sciences, Poznań 60-569, Poland
}

Received June 25, 2014; Accepted May 6, 2015

DOI: $10.3892 / \mathrm{mmr} .2015 .4002$

\begin{abstract}
MicroRNAs (miRNAs/miRs) are a class of short, single-stranded nucleic acids, which have been investigated as potential molecular markers for various types of cancer. The gold-standard and most sensitive method for comparing miRNA levels in cancer tissues is reverse transcription-quantitative polymerase chain reaction (RT-qPCR). This technique uses stably expressed genes for normalisation. The aim of the present study was to improve this model of analysis in the context of RT-qPCR results. A total of six known miRNAs (let-7a, miR-17, miR-27b, miR-125a, miR-125b and miR-206), RNU6B RNA and five mRNAs [erb-b2 receptor tyrosine kinase 2 (ERBB2), hydroxymethylbilane synthase and polymerase (RNA) II (DNA directed) polypeptide A] were analysed pair-wise, in order to determine which biomarker pairs best correlated with the histological groups of 27 breast cancer samples. The lowest P-values and the highest area under the curve values in the receiver operating characteristic analysis were used to select the optimum ratios for discrimination among groups. Among the 21 pairs, miR-17/miR-27b and miR-125a/RNU6B best discriminated three groups of samples with different tumour grades ( $\mathrm{G}$ classification). miR-125b/miR-206 best discriminated two groups of samples with different tumour sizes (pT), let-7a/RNU6B best discriminated two groups of samples with different lymph node status ( $\mathrm{pN}$ ), and let-7a/miR-125b best discriminated groups of samples with negative and positive oestrogen and progesterone receptor status. No pair of miRNAs was found to discriminate well between groups with either a negative or positive human
\end{abstract}

Correspondence to: Dr Tomasz P. Lehmann, Department of Biochemistry and Molecular Biology, University of Medical Sciences, ul. Święcickiego 6, Poznań 60-781, Poland

E-mail: tlehmann@ump.edu.pl

Key words: microRNA, breast cancer, biomarker, normalisation, reverse transcription-quantitative polymerase chain reaction epidermal growth factor receptor 2 (HER2) status. However, one miRNA/mRNA pair, miR-125a/ERBB2, discriminated HER2-negative from HER2-positive groups. The breast cancer samples investigated in the present study were grouped by immunohistological methods into three molecular classes: Luminal, HER2 positive and basal (L, H and B, respectively). In order to discern $\mathrm{L}$ from $\mathrm{H}$ and $\mathrm{L}$ from $\mathrm{B}$, two miRNA pairs were selected: $\mathrm{miR}-125 \mathrm{a} / \mathrm{miR}-125 \mathrm{~b}$ and $\mathrm{miR}-125 \mathrm{a} / \mathrm{miR}-206$. In conclusion, the pair-wise method of RT-qPCR data analysis may be a reasonable alternative to the standard method of using stably expressed reference genes, such as RNU6B RNA, for normalisation. This method may increase the classification power of miRNA biomarkers in breast cancer diagnostics.

\section{Introduction}

MicroRNAs (miRNAs/miRs) provide robustness to biological processes by attenuating the fluctuations in, and overproduction of, mRNA (1). miRNAs have been widely investigated as potential biomarkers for various types of cancer. These small, 19-22 nucleotide-long ribonucleic acids are produced by normal and neoplastic cells. In general, miRNA levels are lower in cancer cells. However, miRNAs that promote tumorigenesis and metastasis, termed oncomirs, are increased in tumour cells (2). Oncomirs, such as miR-17, downregulate the protein synthesis of tumour suppressor genes (3). The majority of genetic mutations that affect miRNAs in cancer, regulate transcription factors or elements of transduction pathway genes (4). Numerous genes encoding miRNAs are methylated in breast cancer cells, which lowers their expression relative to that in healthy cells (5).

The miR, let-7a contains three copies of genes in three genomic loci: 9q22.32 (located 909,430 bp from miR-27b), 11q24.1 (located 46,765 bp from miR-125b 2 in the miR100HG gene) and 22q13.31, a region that is frequently deleted in various types of cancer, including breast cancer (6). The expression of let-7a is lower in invasive ductal carcinomas with lymph node metastasis than in those without lymph node metastasis (7). Let-7a represses the expression of $\mathrm{C}-\mathrm{C}$ chemokine receptor type 7 , by directly targeting its 3 ' untranslated region (UTR) (8). 
Lin28 inhibits the cleavage of pri-let-7 miRNAs, and it has been shown that the ectopic expression of Lin28 completely prevents the processing of pri-let-7a and pri-let-7g (9).

miR-17 was shown to be increased in breast cancer samples compared with samples from healthy individuals (10). Located in 13q31.3, miR-17 is encoded in a cluster (miR17/20 cluster), which contains miR-17, miR-18a, miR-19a/b, miR-20a and miR92a, and is situated in the intron of the C13orf 25 gene $(2,3,11)$. miR-17/20 transcription is stimulated by the cMyc and E2F proteins (12). The expression of all mature miRNA from the genomic location of the miR-17/20 cluster, correlates with the copy number of the gene in breast cancer (2). The miR-17/20 cluster functions as a tumour suppressor in human breast cancer by decreasing amplified in breast cancer I and cyclin Dl expression $(13,14)$. miR-17 has been demonstrated to be increased in breast cancer, and to exert tumour-suppressive and anti-invasive functions in breast cancer cells $(2,13,15)$.

miR-27b is located in $9 \mathrm{q} 22.32$ in the intron of $C 9$ orf3. It is located between miR-23b and miR-24-1, and is close to the PHD finger protein 2 and Fanconi anemia, complementation group $\mathrm{C}$ genes, which are associated with an increased risk of breast cancer (16). miR-27b-3p is homologous to miR-27a-3p and interacts with the 3'UTR of cytochrome P450 1B1 (CYP1BI) mRNA, with which it shares 19 bases. The product of this gene is increased in a number of types of cancer, and is involved in the metabolism of pro-carcinogens (17). The gene product of erb-b2 receptor tyrosine kinase 2 (ERBB2), also termed HER2, as well as those of epidermal growth factor and tumour necrosis factor- $\alpha$, promotes miR-27b expression through the protein kinase $\mathrm{B} /$ nuclear factor- $\kappa \mathrm{B}$ signalling cascade (18).

In humans, three copies of miRNA-125 sequence, miR-125a, miR-125b1 and miR-125b2, are located in chromosomes 19, 11 and 21, respectively. The genes encoding miR-125b, which is downregulated in breast cancer, are located at two sites: One in chromosome 11q24.1 (the region frequently deleted in breast cancer) and one in chromosome 21q21.1 (19,20). Numerous genes that regulate proliferation and apoptosis in mammary cells are targeted by miR-125a and miR-125b $(21,22)$. miR-125b suppresses tumour growth by diminishing the expression of oncoproteins, such as ERBB2 and ERBB3 (23). Cancer cells overexpressing miR-125b exhibit growth inhibition and a reduced migratory and invasive capacity (23). Hypermethylation of the miR-125b promoter has been observed to partially account for the reduction of miR-125b expression in human breast cancer cells (24). miR-125a expression is also decreased in breast cancer. It is located in $19 \mathrm{q} 13.41$, in close proximity to a region that is associated with breast cancer (19q13.3) (19,25).

miR-206 and miR-133b form a cluster in an intergenic region of chromosome $6 \mathrm{p} 12.2$ (26). miR-206 regulates Estrogen receptor 1 (ESRl) gene expression (27). Exogenous miR-206 inhibits the proliferation of various cell types, through the induction of cell cycle arrest and apoptosis via its target genes NOTCH3 (28), MET (29) and cyclin D2 (30).

There are a number of variables in reverse transcription-quantitative polymerase chain reaction (RT-qPCR) quantification of miRNAs, which may cause variability within the same sample and among different samples within a group. The method used to overcome these variations is termed normalisation, which aims to diminish artefacts introduced during the experimental procedure. The gold standard in RT-qPCR is to use internal reference RNA (31). The most popular normalisation method for miRNA analysis, is the application of RNU6B RNA as an internal reference, which is recommended by Applied Biosystems (31). The method relies on calculating the difference in cycle threshold $(\Delta \mathrm{Ct})$ between a target miRNA $\mathrm{Ct}$ and reference $\mathrm{Ct}$ (RNU6B). It is recommended that $\Delta \mathrm{Ct}$ (indicated also by the target : reference ratio, if $\mathrm{Ct}$ were converted into relative quantities) should be characterised by the lowest variation for an investigated group of samples and could be compared with the $\Delta \mathrm{Ct}$ (ratio) of the control group (31). A disadvantage of this method is the occurrence of high variance of the mean $\Delta \mathrm{Ct}$. This situation is particularly problematic for samples, in which the expression of the target gene is inversely correlated with the expression of the selected reference gene. The approach used by this group to improve the model is to obtain a more constant $\Delta \mathrm{Ct}$ (target : reference ratio). This hypothesis is similar to 'repeated pairwise correlation and regression analysis', as developed by Matthaei et al (32) following proposal by Pfaffl et al (33).

In the present study, six miRNAs were selected (hsa-let-7a-5p, hsa-miR-17-3p*, hsa-miR-27b-5p*, hsa-miR-125a-3p*, hsa-miR-125b-1-3p* and hsa-miR-206), which are known regulators of oncogenesis, particularly in breast cancer. Each of the selected miRNAs had previously been investigated in terms of their expression in breast cancer cells. The aim of the present study was to confirm their potential as biomarkers for differentiating breast cancer types. This approach may help to identify biomarkers with an increased specificity for the molecular classification of breast cancer, as miRNAs are more stable in archival samples and miRNA profiling is more reliable than mRNA profiling to determine biomarkers for poor-quality samples.

It was hypothesised that through the calculation of all possible ratios ('diffpairs') between six miRNAs and RNU6B RNA, the optimal RNA marker pairs for breast cancer classification, corresponding to immunohistochemical criteria, would be determined. In order to achieve this, the correction method was applied to diminish the variability in the ratios of two selected miRNAs. In the present study, a small group of breast tumour samples were used to assess an improved 'diffpairs' model of RT-qPCR data analysis, thereby increasing the diagnostic value of miRNA assays conducted using RT-qPCR.

\section{Patients and methods}

Patients and samples. Tissue samples were obtained from 27 patients who had not undergone preoperative radiotherapy or chemotherapy, and had received breast cancer surgery at the Department of Surgery, Chair and Clinic of Oncology of Poznań University of Medical Sciences (Poznań, Poland). The classification of these patients is shown in Table I. The study design was approved by the University Bioethical Board of Poznań University of Medical Sciences. The tumour tissue samples and blood samples for comprehensive experiments were collected after written informed consent had been obtained from all participants. Following surgery, the breast tumour samples were divided into two groups: One group (21 samples) was stored in liquid nitrogen and the second group (6 samples) was formalin-fixed and paraffin-embedded 
(FFPE). The patient samples were classified according to the TNM classification of tumours (34). Prior to RNA extraction, corresponding haematoxylin and eosin-stained tumour tissue sections were created, and the percentage of cancer cells in these sections was evaluated. In the present study, the average percentage of tumour cells per section was $76 \%$.

RNA extraction. miRNA and mRNA were extracted from frozen tissue using the mirVana ${ }^{\mathrm{TM}}$ miRNA isolation kit (Life Technologies, Carlsbad, CA, USA). Total RNA from paraffin-embedded tissues was extracted using a RecoverAll ${ }^{\mathrm{TM}}$ total nucleic acid isolation kit (Life Technologies). The quantity of nucleic acid obtained, was assessed using the micro-spectrophotometer Nano-100 (Hangzhou Allsheng Instruments CO., Ltd., Hangzhou, China).

$R T-q P C R$. In order to evaluate RNA expression levels, TaqMan microRNA assays (Life Technologies) for RT-qPCR were conducted for hsa-let-7a-5p (000377), hsa-miR-17-3p* (002421), hsa-miR-27b-5p* (002174), hsa-miR-125a-3p* (002199), hsa-miR-125b-1-3p* (002378), hsa-miR-206 (000510) and RNU6B (001093). All samples were reverse transcribed using the TaqMan microRNA reverse transcription kit and specific primers from the TaqMan microRNA assay (Life Technologies). Briefly, the following reaction conditions of RT were used according to the manufacturer' instructions: $10 \mathrm{mM}$ dNTP, $5 \mathrm{U} / \mu 1$ MultiScribe reverse transcriptase, $2 \mathrm{U} / \mu 1$ RNase inhibitor, $5 \mu 1$ total RNA, $3 \mu 1$ specific reverse transcription primer; $30 \mathrm{~min}$ at $16^{\circ} \mathrm{C}, 30 \mathrm{~min}$ at $42^{\circ} \mathrm{C}$ then $5 \mathrm{~min}$ at $85^{\circ} \mathrm{C}$. TaqMan Universal PCR master mix and specific primers from the TaqMan microRNA assays were used to quantify samples, using a Roche LC 480 cycler (Roche Diagnostics, Basel, Switzerland). Briefly, the following reaction conditions of PCR were applied according to the manufacturer's instructions: $0.5 \mu 1$ TaqMan microRNA assay primers, $1 \mu \mathrm{l}$ RT reaction product, $5 \mu \mathrm{l}$ TaqMan $2 \mathrm{X}$ Universal PCR master mix, No AmpErase UNG; $10 \mathrm{~min}$ at $95^{\circ} \mathrm{C}, 45$ cycles of $15 \mathrm{sec}$ at $95^{\circ} \mathrm{C}$ and $1 \mathrm{~min}$ at $60^{\circ} \mathrm{C}$. The relative quantities of all miRNAs were calculated using a standard curve for miR-206. All samples were calculated according to the miR-206 efficiency as the supplier (Life Technologies) has standardised their miRNA assays to obtain a similar amplification efficiency.

The ERBB2 (Hs01001580_m1), ESR1 (Hs00174860_m1) and PGR (Hs01556702_m1) mRNA levels were analysed by RT and TaqMan qPCR (Life Technologies). Hydroxymethylbilane synthase (HMBS; Hs00609293_g1) and POLR2A (Hs00172187_m1) were used as reference mRNA levels.

Statistical analysis. Statistical analysis was performed using the Microsoft Excel 2003 (Microsoft Corporation, Redmond, WA, USA), Instat (GraphPad InStat version 3.05 for Windows 95, GraphPad Software, San Diego, CA, USA), Statistica 10 version 10 (StatSoft, Inc., Tulsa, OK, USA) and GeNorme software programs (Ghent University Hospital Center for Medical Genetics, Ghent, Belgium). $\mathrm{P}<0.05$ was considered to indicate a statistically significant difference. The Mann-Whitney (MW) and Kruskal-Wallis tests were used to compare the differences in the mean values of relative expression levels between groups of samples, obtained from the RT-qPCR experiments. The means of the $\log _{10}$ ratios
Table I. Classification of patient groups using the TNM system and expression of the ER, PR and HER2 receptors.

\begin{tabular}{lcc}
\hline Type & Number of patients & Percentage \\
\hline G1 & 5 & 19.2 \\
G2 & 12 & 46.2 \\
G3 & 9 & 34.6 \\
pT1 & 13 & 50.0 \\
pT2 & 12 & 46.2 \\
pT3 & 0 & 0.0 \\
pT4 & 1 & 3.8 \\
pN0 & 14 & 53.8 \\
pN1 & 11 & 42.3 \\
pN2 & 1 & 3.8 \\
ER (-) & 8 & 30.8 \\
ER (+) & 18 & 69.2 \\
PR (-) & 9 & 34.6 \\
PR (+) & 17 & 65.4 \\
HER2 (-) & 20 & 76.9 \\
HER2 (+) & 6 & 23.1 \\
\hline
\end{tabular}

ER, estrogen receptor; PR, progesterone receptor; HER2, human epidermal growth factor receptor 2 .

between the target gene expression levels and the 'reference' gene expression levels were also calculated.

The correlation between each of the 21 pairs of 7 RNAs for 16 groups of histologically-defined samples was estimated. A total of 336 correlation coefficients (R) were obtained, which were positive or negative. The results were calculated using the following algorithm: i) All crossing point $(\mathrm{Ct})$ values obtained from the RT-qPCR analysis of 7 miRNAs were transformed to the relative concentrations based on a standard curve of miR-206 using the LC 480 software 1.5.0.39 (Roche Diagnostics). ii) In each histological group, the correlation coefficients and linear regressions were calculated for the relative concentrations of a pair of miRNAs or RNU6B RNA (for all 21 combinations of 7 RNAs, including RNU6B). When $\mathrm{R}$ was positive, the mean ratios of the target and 'reference' miRNAs were calculated $\left(\mathrm{X}_{\mathrm{n} \text { tar }} / \mathrm{Y}_{\mathrm{m} \text { ref }}\right)$. iii) A negative correlation of the target $(\mathrm{X})$ and 'reference' (Y) RNAs implied a requirement for recalculating the target and 'reference' ratios. The regression line and its formula were calculated, and the $\mathrm{X}_{\mathrm{tar} 0}$ crossing point was established $\left(Y_{r e f}=0\right)$. The difference $X_{\operatorname{tar} 0}-X_{n \text { tarn }}$ for each target value was calculated. Subsequently, the ratios $\left(\mathrm{X}_{\operatorname{tar} 0}-\mathrm{X}_{\mathrm{n} \operatorname{tarn}}\right) / \mathrm{Y}_{\mathrm{m} \text { ref }}$ for each sample and their means were calculated. iv) Mean values of two groups of samples were subsequently compared using a non-parametric method (Mann-Whitney). Using this approach, lower P-values were obtained by comparing the target-reference ratios without correcting for negative correlations in the groups.

A similar approach was used for miRNA and mRNA, which were transformed to the relative concentrations based on standard curves (s.c.; miR-206 s.c. for all miRNAs and specific s.c. for 5 mRNAs). The correlation coefficients and linear regressions were subsequently calculated for each histological group. When the correlation was positive, the mean miRNA and mRNA ratios were calculated $\left(\mathrm{X}_{\mathrm{n} \text { miRNA }} / \mathrm{Y}_{\mathrm{mmRNA}}\right)$. A negative correlation between the miRNA and mRNA required recalculation of the ratio. The regression line and its formula were calculated and the $\mathrm{X}_{0 \text { miRNA }}$ crossing point was established 

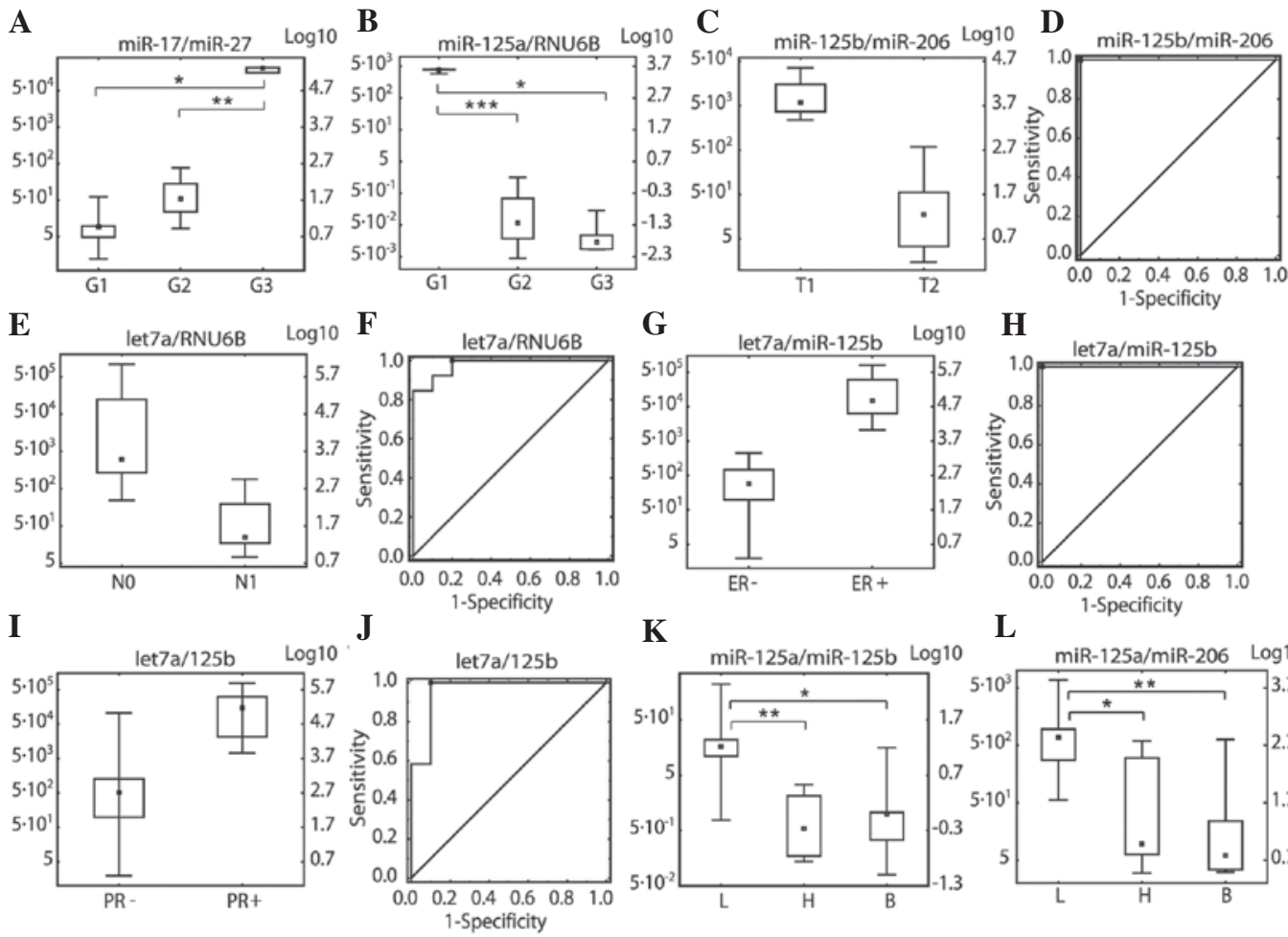

$\mathbf{K}$

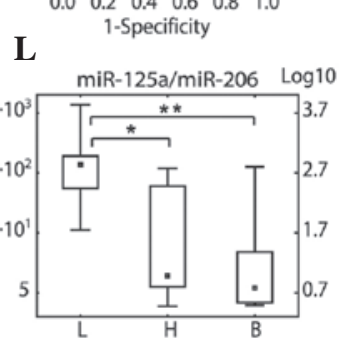

Figure 1. Relative expression levels expressed as miRNA/miRNA ratios. (A) miR-17/miR-27b in three grading groups (G1-G3), (B) miR-125a/RNU6B in three grading groups (G1-G3), (C) miR-125b/miR-206 in tumour size groups (pT1 and pT2), (D) miR-125b/miR-206 ROC in tumour size groups (pT1 and pT2), (E) let7a/RNU6B in lymph node metastasis status groups ( $\mathrm{pN0}$ and pN1), (F) let7a/RNU6B ROC analysis in lymph node status groups (pN0 and pN1), (G) let7a/miR-125b ER status (ER- and ER+), (H) let7a/miR-125b ROC analysis in ER status groups (ER- and ER+), (I) let7a/miR-125b PR status (PR- and $\mathrm{PR}+$ ), (J) let7a/miR-125b ROC analysis in PR status groups (PR- and PR+), (K) miR-125a/miR-125b in three molecular classification groups (L, luminal; B , basal; H, HER2 positive) (L) miR-125a/miR-206 in three molecular classification groups. ${ }^{*} \mathrm{P}<0.05,{ }^{* *} \mathrm{P}<0.01$ and ${ }^{* * *} \mathrm{P}<0.001$. miR/miRNA, microRNA; ROC, receiver operating characteristic; ER, estrogen receptor; PR, progesterone receptor.

$\left(\mathrm{Y}_{\mathrm{mRNA}}=0\right)$. The difference $\left(\mathrm{X}_{0 \text { miRNA }}-\mathrm{X}_{\mathrm{n} \text { miRNA }}\right)$ in each miRNA value was calculated. The ratio $\left(\mathrm{X}_{0 \text { miRNA }}-\mathrm{Xn}_{\text {miRNA }}\right) / \mathrm{Ym}$ mRNA for each sample and its mean was subsequently calculated. The mean values of the two groups of samples were then compared.

Receiver operating characteristic (ROC) analyses were performed for all pairs of miRNA/miRNA, miRNA/RNU6B and miRNA/mRNA. The lowest P-values and the highest area under the curve (AUC) values were used to select the best ratios for potential biomarkers with the highest specificity and sensitivity.

\section{Results}

miRNA ratios. In the present study, an attempt was made to establish a modified approach for identifying molecular markers of breast cancer. Breast cancer samples were classified according to tumour grade (G1-G3 according to Elston-Ellis classification, also termed the Nottingham classification) (35); the TNM classification (Tumour size, pT1 or pT2; lymph node metastasis, pN0 or pN1); receptors status [Estrogen receptor (ER) status (ER-, ER+), progesterone receptor (PR) status (PR-, PR+) and human epidermal growth factor receptor 2 (HER2) status] (Table I).

The miRNAs, let-7a, miR-17, miR-27b, miR-125a, miR-125b and miR-206 were evaluated in breast cancer samples. In breast cancer cells, these miRNAs have been classified as tumour suppressive (36). RNU6B (001093) was selected as the reference for miRNA quantification.
The GeNorme software was used to rank the potential reference miRNAs, and miR-125a, miR-125b and RNU6B were the most stable of the samples. The average expression stability for each miRNA was: miR-125a, 0.115; miR-125b-1, 0.119; RNU6B, 0.125; let-7a, 0.206; miR-17, 2.544; miR-27b, 3.959; and miR-206, 29.536. The uncertainty over reference miRNA expression prompted the investigation for an alternative calculation model enabling a reliable miRNA expression analysis.

All combinations of ratios for let-7a, miR-17, miR-27b, miR-125a, miR-125b-1, miR-206 and RNU6B were calculated for each group of samples (G, pT, pN, ER, PR, HER2 and molecular group). The results are presented in Table II. Calculations were performed using an algorithm that was executed by macros in Microsoft Excel. Any outliers were eliminated.

For the tumour grade ( $\mathrm{G}$ classification), which contains three classes of samples, the following combinations of miRNAs were selected: $\mathrm{miR}-17 / \mathrm{miR}-27 \mathrm{~b}$ to discriminate grades 2 and 3 and miR-125a/RNU6B to discriminate grades 1 and 3 or 1 and 2 . These miRNAs were selected based on P-values and the consequent increase or decrease in their ratios from $\mathrm{G} 1$ to $\mathrm{G} 3$ (Fig. 1A and B).

For pT, pN, ER, PR and HER2, the P-values were calculated and ROC analyses were performed. The lowest P-values and the highest AUC values were used to select the best ratios (Fig. 1C-J; Table II and III). The following ratios were selected: miR-125b/miR-206 for $\mathrm{pT}$, let-7a/RNU6B for $\mathrm{pN}$, let-7a/miR-125b for ER and let-7a/miR-125b for PR. No suitable pair of miRNA/miRNA was found for HER2. 


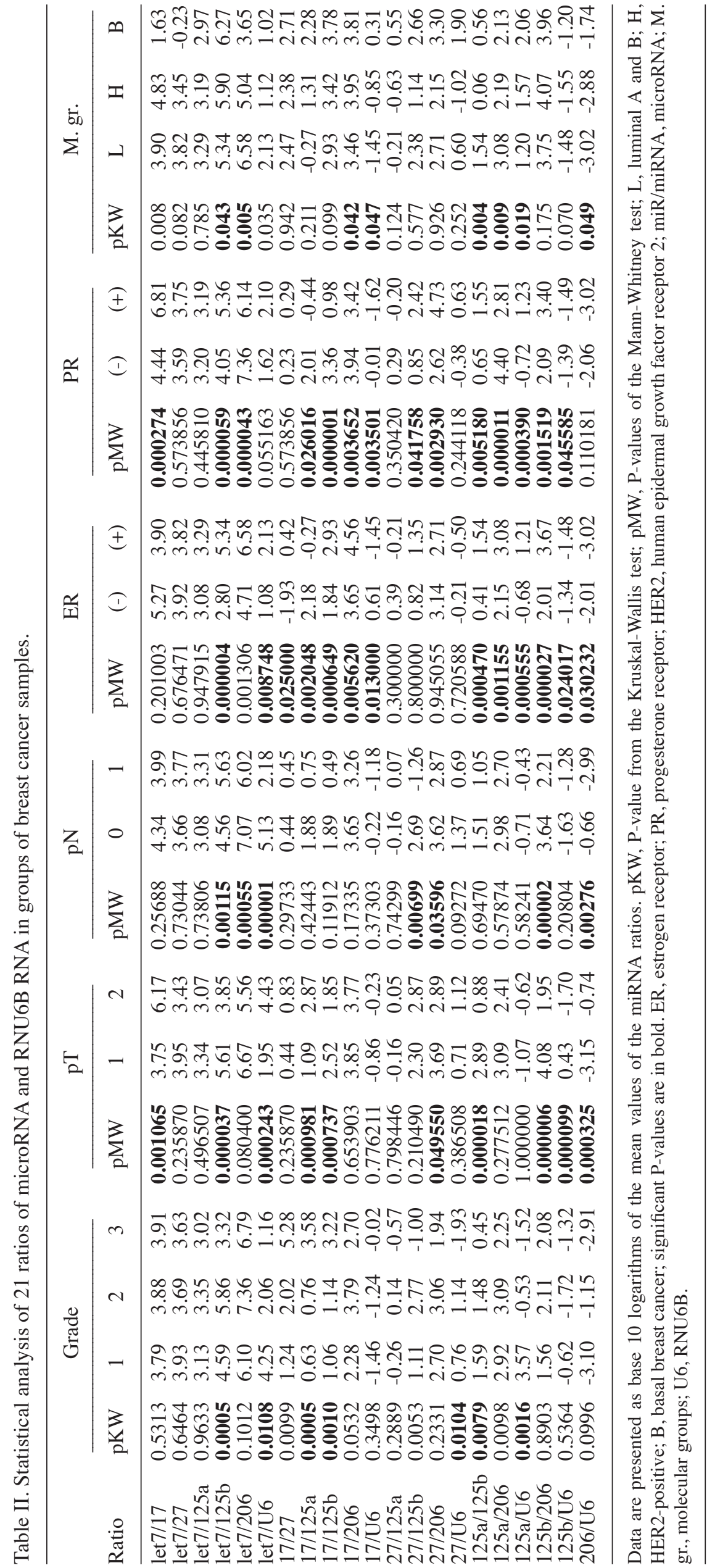



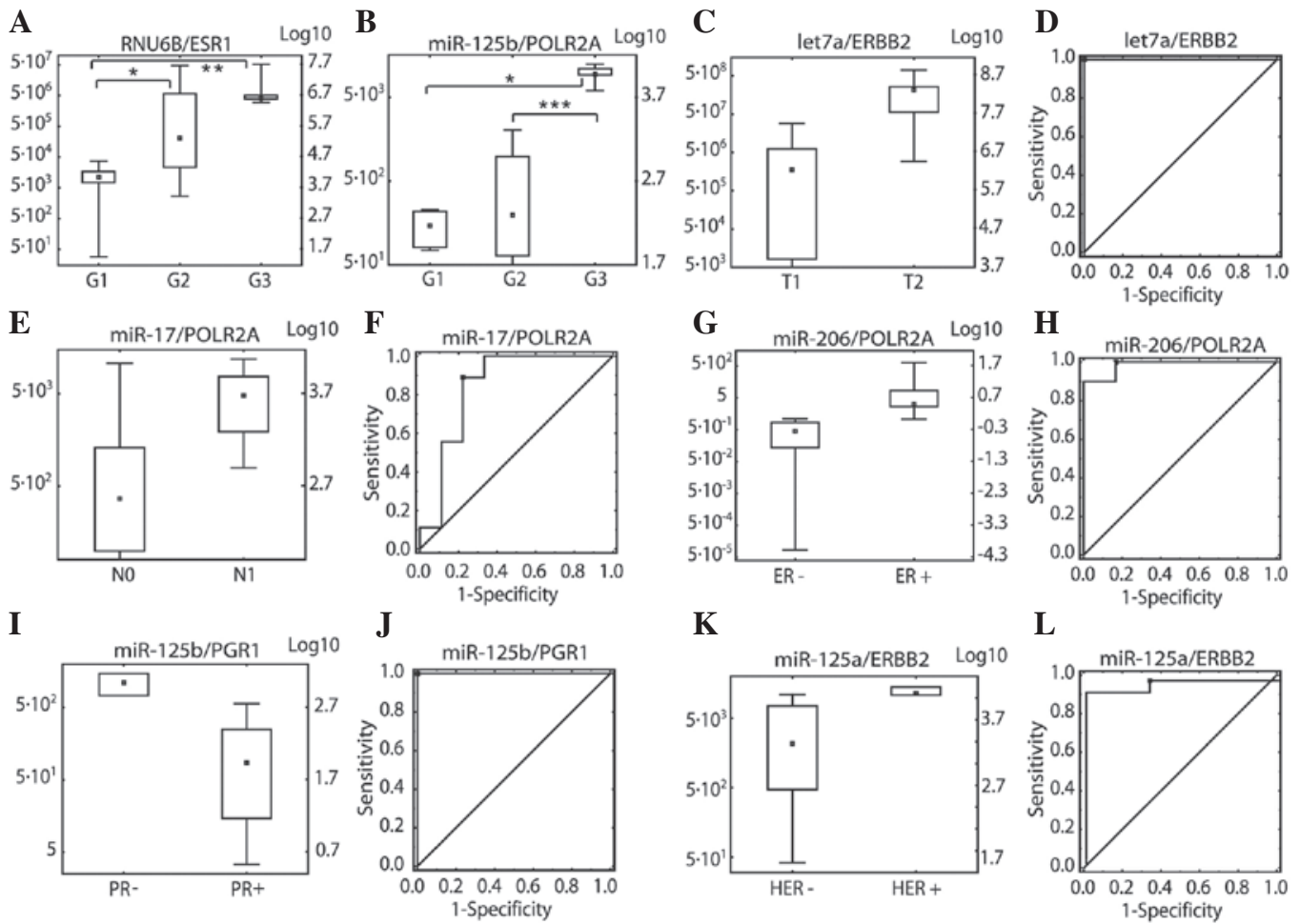

Figure 2. Relative expression levels expressed as miRNA/mRNA ratios. (A) RNU6B/ESR1 in three grading groups (G1-G3), (B) miR-125b/POLR2A in three grading groups (G1-G3), (C) let7a/ERBB2 in tumour size groups (pT1 and pT2), (D) let7a/ERBB2 ROC in tumour size groups (pT1 and pT2), (E) miR-17/POLR2A in lymph node status groups ( $\mathrm{pN0}$ and $\mathrm{pN1}$ ), (F) miR-17/POLR2A ROC analysis in lymph node metastasis status groups (pN0 and $\mathrm{pN1}$ ), (G) miR-206/POLR2A ER status (ER- and ER+), (H) miR-206/POLR2A ROC analysis in ER status groups (ER- and ER+), (I) miR-125b/PGR PR status (PR- and PR+), (J) miR-125b/PGR ROC analysis in PR status groups (PR- and PR+), (K) miR-125a/ERBB2 in three HER2 receptor status groups (HER-and HER+), (L) miR-125a/ERBB2 in three molecular classification groups. ${ }^{*} \mathrm{P}<0.05,{ }^{* * *} \mathrm{P}<0.01$ and ${ }^{* * * *} \mathrm{P}<0.001$. miR/miRNA, microRNA; ROC, Receiver operating characteristic; ER, estrogen receptor; PR, progesterone receptor; HER2, human epidermal growth factor receptor 2.

The breast cancer samples investigated in the present study were grouped into three molecular classes by immunohistological methods: Luminal, HER2 positive and basal (L, H, B; Fig. $1 \mathrm{~K}$ and $\mathrm{L}$ ). In order to discern $\mathrm{L}$ from $\mathrm{H}$, and $\mathrm{L}$ from $\mathrm{B}$, the two miRNA ratios selected based on the lowest P-values were miR-125a/miR-125b and miR-125a/miR-206, respectively.

In conclusion, a total of 5 miRNAs (let-7, 125a, 125b, 206 and RNU6B) were selected, which were able to discriminate tumour grade, $\mathrm{pT}, \mathrm{pN}, \mathrm{ER}, \mathrm{PR}$ and three molecular groups in breast cancer samples.

miRNA/mRNA ratios. As PR, ER and HER2 are the primary markers used in the immunohistochemical classification of breast cancer samples, the mRNA levels of PGR, ESR 1 and $E R B B 2$, and of two reference genes, HMBS and POLR $2 A$, were determined in the breast cancer samples. A ratio for each receptor mRNA (PGR, ESR1 and ERBB2) was calculated using POLR2A. Receptor mRNA levels were correlated with their protein levels in the histological groups, PR, ER and HER2, respectively. P-values for ESRI and $P G R$ were calculated using a non-parametric Mann-Whitney test (samples were not considered to have a normal distribution). P-values for ERBB2 were calculated using a t-test (sample was considered to have a normal distribution).

The ratios of each miRNA to $P G R, E S R 1, E R B B 2, H M B S$ and POLR2A mRNA were calculated for each group (Table II). In accordance with the results observed for the ratio of miRNA/miRNA, the correlation in a single group may be positive or negative. The results were therefore calculated using the same algorithm as that, which was used for miRNA/miRNA.

For the tumour grade (G classification), which contained three classes of samples, the following combinations of miRNA/mRNA were selected: RNU6B/ESR 1 to discriminate grades 1 and 2, and grades 1 and 3, and miR-125b/POLR2A to discriminate grades 2 and 3 . These miRNAs were selected based on the P-values that were calculated using the Kruskal-Wallis test and subsequently, for each pair (1 with 2, 2 with 3 and 1 with 3), using the Mann-Whitney test (Fig. 2A and B).

For pT, pN, ER, PR and HER2, P-values were calculated and ROC analyses were performed. The lowest P-values and the highest AUC values were used to select the best ratios (Fig. 2C-L; Table IV and V). The following ratios were selected: let-7a/ERBB2 for $\mathrm{pT}, \mathrm{miR}-17 / P O L R 2 A$ for $\mathrm{pN}$, miR-206/POLR $2 A$ for ER, miR-125b/PGR for PR and miR-125a/ERBB2 for HER2.

Due to the poor amplification of cDNA from six of the FFPE samples, the results were insufficient to discern the three molecular classes of breast cancer using the miRNA/mRNA ratios. However, the miRNA/mRNA ratio did allow the discrimination of HER2-negative groups $(0,1,2)$ and positive groups (3) using miR-125a/ERBB2.

In conclusion, the strategy of using the miRNA/mRNA ratio requires the analysis of $5 \mathrm{miRNAs}$ (let-7a, miR-17, miR-125b, miR-206, RNU6B) and 4 mRNAs (ESR1, PGR, ERBB2, POLR2A). An advantage of the miRNA/mRNA ratio is that it allowed the discrimination of HER2-negative groups $(0,1,2)$ and positive groups (3) using the miR-125a/ERBB2 ratio. 
Table III. Receiver operating characteristic analysis of 21 ratios of microRNA and RNU6B RNA 21 ratios and groups of breast cancer samples.

A, Tumour size grouping

\begin{tabular}{lllll}
\hline Pairing & AUC & SE & Fidelity & P-value \\
\hline let7/17 & 0.879 & 0.077 & 0.728 & 0.001065 \\
let7/125b & 0.967 & 0.033 & 0.902 & 0.000037 \\
let7/U6 & 0.923 & 0.054 & 0.817 & 0.000243 \\
$17 / 125 \mathrm{a}$ & 0.886 & 0.075 & 0.739 & 0.000981 \\
$17 / 125 \mathrm{~b}$ & 0.894 & 0.082 & 0.733 & 0.000737 \\
$27 / 206$ & 0.910 & 0.070 & 0.772 & 0.049550 \\
$125 \mathrm{a} / 125 \mathrm{~b}$ & 0.939 & 0.055 & 0.832 & 0.000018 \\
$125 \mathrm{~b} / 206$ & 1.000 & 0.000 & 1.000 & 0.000006 \\
$125 \mathrm{~b} / \mathrm{U} 6$ & 0.970 & 0.033 & 0.904 & 0.000099 \\
$206 / \mathrm{U} 6$ & 0.815 & 0.129 & 0.562 & 0.000325 \\
\hline
\end{tabular}

B, Lymph node status

\begin{tabular}{lcccr}
\hline Pairing & AUC & SE & Fidelity & P-value \\
\hline let7/125b & 0.885 & 0.071 & 0.746 & 0.001145 \\
let7/206 & 1.000 & 0.025 & 1.000 & 0.000550 \\
let7/U6 & 0.977 & 0.111 & 0.928 & 0.000012 \\
$27 / 125 b$ & 0.883 & 0.108 & 0.666 & 0.006993 \\
$27 / 206$ & 0.833 & 0.024 & 0.621 & 0.035964 \\
$125 \mathrm{~b} / 206$ & 0.982 & 0.076 & 0.736 & 0.000023 \\
$206 / \mathrm{U} 6$ & 0.882 & & & 0.002761 \\
\hline
\end{tabular}

C, Oestrogen receptor status

\begin{tabular}{lllll}
\hline Pairing & AUC & SE & Fidelity & P-value \\
\hline let7/125b & 1.000 & & 1.000 & 0.000004 \\
let7/206 & 0.864 & 0.093 & 0.682 & 0.001306 \\
let7/U6 & 0.828 & 0.084 & 0.663 & 0.008748 \\
$17 / 27$ & 0.923 & 0.074 & 0.778 & 0.025000 \\
$17 / 125 \mathrm{a}$ & 0.873 & 0.105 & 0.667 & 0.002048 \\
$17 / 125 \mathrm{~b}$ & 0.905 & 0.063 & 0.689 & 0.000649 \\
$17 / 206$ & 0.852 & 0.083 & 0.574 & 0.013620 \\
$17 / \mathrm{U} 6$ & 0.817 & 0.061 & 0.793 & 0.000470 \\
$125 \mathrm{a} / 125 \mathrm{~b}$ & 0.913 & 0.068 & 0.775 & 0.001155 \\
$125 \mathrm{a} / 206$ & 0.909 & 0.081 & 0.759 & 0.000555 \\
$125 \mathrm{a} / \mathrm{U} 6$ & 0.917 & 0.024 & 0.934 & 0.000027 \\
$125 \mathrm{~b} / 206$ & 0.981 & 0.100 & 0.598 & 0.024017 \\
$125 \mathrm{~b} / \mathrm{U} 6$ & 0.795 & 0.115 & 0.030232 \\
206/U6 & 0.755 & & \\
\hline
\end{tabular}

D, Progesterone receptor status

\begin{tabular}{llccc}
\hline Pairing & AUC & SE & Fidelity & P-value \\
\hline let7/17 & 0.908 & 0.088 & 0.735 & 0.000274 \\
let7/125b & 0.958 & 0.044 & 0.872 & 0.000059 \\
let7/206 & 1.000 & 0.117 & 1.000 & 0.541 \\
$17 / 125 \mathrm{a}$ & 0.771 & & 1.000 & 0.0260043 \\
$17 / 125 \mathrm{~b}$ & 1.000 & 0.084 & 0.698 & 0.000001 \\
$17 / 206$ & 0.864 & 0.108 & 0.629 & 0.003652 \\
$17 / \mathrm{U6}$ & 0.840 & 0.097 & 0.665 & 0.003501 \\
$27 / 125 \mathrm{~b}$ & 0.854 & 0.035 & 0.909 & 0.002930 \\
$27 / 206$ & 0.977 & 0.090 & 0.662 & 0.005180 \\
$125 \mathrm{a} / 125 \mathrm{~b}$ & 0.838 & 0.075 & 0.776 & 0.000011 \\
$125 \mathrm{a} / 206$ & 1.000 & 0.084 & 0.719 & 0.000390 \\
$125 \mathrm{a} / \mathrm{U} 6$ & 0.923 & 0.084 & 0.719 & 0.001519 \\
$125 \mathrm{~b} / 206$ & 0.883 & 0.045585 \\
$125 \mathrm{~b} / \mathrm{U} 6$ & 0.883 & & & \\
\hline
\end{tabular}

Only ratios with significant $\mathrm{P}$-values are shown. AUC, area under the curve; SE, standard error; U6, RNU6B. 


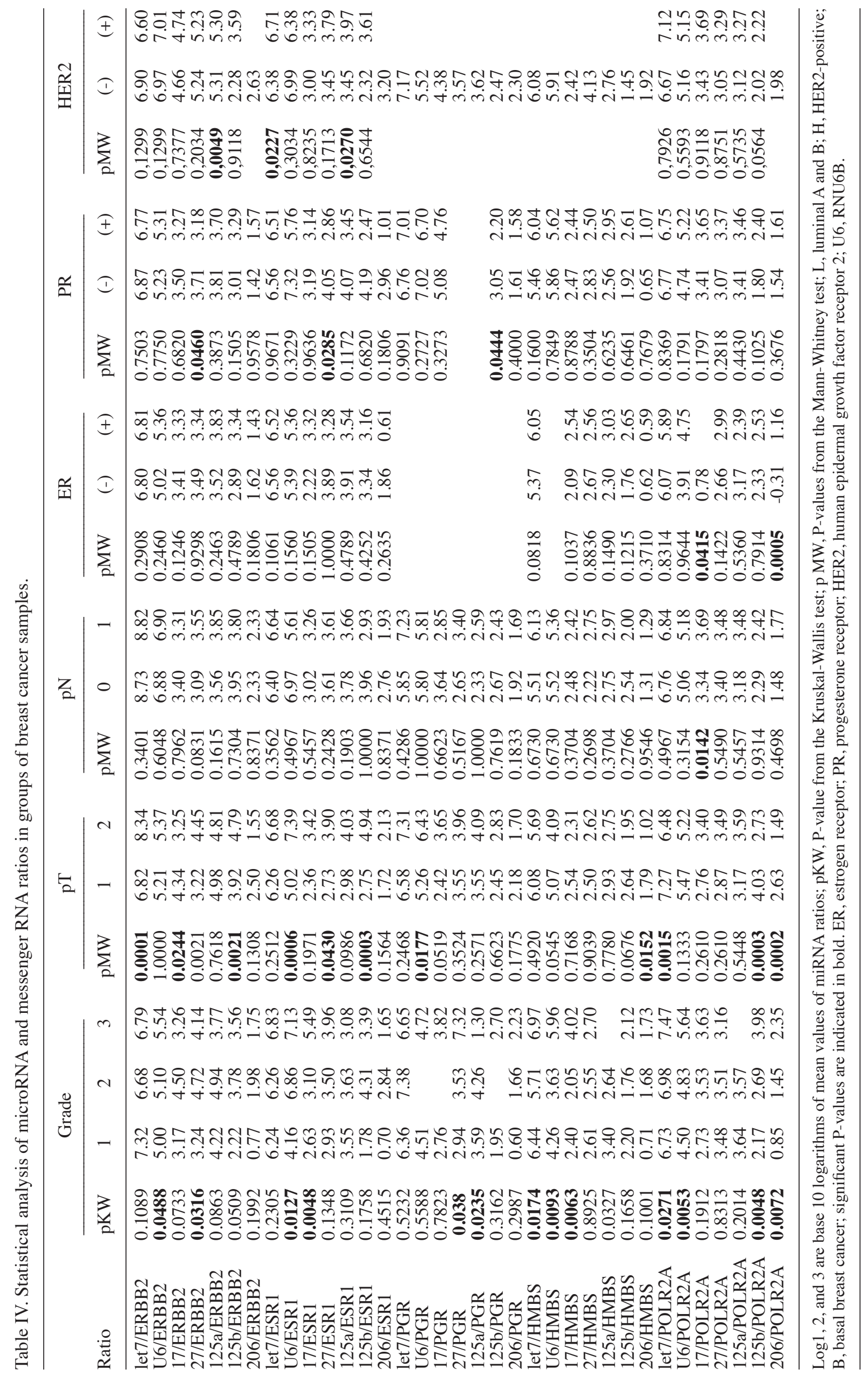


Table V. ROC analysis of microRNA:mRNA ratios.

A, Tumour size grouping

\begin{tabular}{lllll}
\hline miR/mRNA & AUC & SE & Fidelity & P-value \\
\hline let7/ERBB2 & 1.000 & & 1.000 & 0.0001 \\
17/ERBB2 & 0.815 & 0.103 & 0.614 & 0.0244 \\
27/ERBB2 & 0.869 & 0.088 & 0.696 & 0.0021 \\
125b/ERBB2 & 0.913 & 0.069 & 0.777 & 0.0021 \\
RNU6B/ESR1 & 0.88 & 0.093 & 0.697 & 0.0006 \\
27/ESR1 & 0.759 & 0.114 & 0.536 & 0.0430 \\
125b/ESR1 & 0.963 & 0.043 & 0.878 & 0.0003 \\
RNU6B/PGR & 0.571 & 0.187 & 0.205 & 0.0177 \\
206/HMBS & 0.847 & 0.1 & 0.652 & 0.0152 \\
let7/POLR2A & 1.000 & & 1.000 & 0.0015 \\
125b/POLR2A & 0.963 & 0.043 & 0.879 & 0.0003 \\
206/POLR2A & 1.000 & & 1.000 & 0.0002 \\
& & & & \\
\hline
\end{tabular}

B, Lymph node status

\begin{tabular}{lcccc}
\hline miR/mRNA & AUC & SE & Fidelity & P-value \\
\hline 17/POLR2A & 0.84 & 0.105 & 0.635 & 0.0142 \\
\hline
\end{tabular}

C, Oestrogen receptor status

\begin{tabular}{lcccc}
\hline miR/mRNA & AUC & SE & Fidelity & P-value \\
\hline 17/POLR2A & 0.806 & 0.133 & 0.544 & 0.0415 \\
206/POLR2A & 0.983 & 0.026 & 0.932 & 0.0005
\end{tabular}

D, Progesterone receptor status

\begin{tabular}{lcccc}
\hline miR/mRNA & AUC & SE & Fidelity & P-value \\
\hline 27/ERBB2 & 0.815 & 0.104 & 0.611 & 0.0460 \\
27/ESR1 & 0.821 & 0.141 & 0.545 & 0.0285 \\
125b/PGR & 1.000 & & 1.000 & 0.0444 \\
\hline
\end{tabular}

E, Human epithelial growth factor receptor 2 status

\begin{tabular}{lcccr}
\hline $\mathrm{miR} / \mathrm{mRNA}$ & AUC & SE & Fidelity & P-value \\
\hline 125a/ERBB2 & 0.978 & 0.033 & 0.913 & 1.0000 \\
let7/ESR1 & 1.000 & & 1.000 & 1.0000 \\
125a/ESR1 & 0.911 & 0.088 & 0.739 & 1.0000 \\
\hline
\end{tabular}

All pairs of miRNA and RNU6B RNA were analysed using ROC analysis to estimate the specificity and sensitivity of the biomarker pairs. Only ratios with significant P-values are shown. ROC, receiver operating characteristic; AUC, area under the curve; SE, standard error; $\mathrm{miR} / \mathrm{miRNA}$, microRNA.

\section{Discussion}

In the present study, a total of 8 pairs of miRNA:miRNA and miRNA:RNU6B, and one miRNA:mRNA pair were identified, which may be used as markers in order to improve the classification of breast cancer samples. These markers may aid in the classification of the cancer grade, tumour size (pT), lymph node metastasis (pN), ER status, PR status, and three molecular breast cancer types (luminal, HER2 positive and basal). In addition, pairs of miRNA:mRNA were identified, which were able to distinguish HER2 receptor groups. To achieve this, the 'diffpair' method was utilised, and the correction method to diminish the variability in the ratios of two selected miRNAs was applied.

Statistical criteria enabled the selection of 8 of the 21 possible pairings of 7 types of RNA. In the set of 21 pairs, each miRNA and RNU6B pair was presented only once; inverted pairs were not assessed. In order to achieve greater accuracy and lower P-values, the correction method was applied when calculating the means of all the RNA pairs in the groups. This improved the significance in the discrimination of groups of samples. The samples were grouped according to six criteria (grade, Tp, Np, ER status, PR status, HER2 status and molecular type). These six criteria were used to divide the samples into 16 groups. Based on the present results, it was hypothesised that measuring miR-17/miR-27b to discriminate grades G2 and G3, miR-125a/RNU6B to discriminate grades G1 and G3 and grades G1 and G2, miR-125b/miR-206 to assess tumour size (pT), let-7a/RNU6B to assess lymph node metastasis, and let-7a/miR-125b to determine the ER and PR status may be effective. Furthermore, it was hypothesised that HER2 negative $(0,1,2)$ and positive $(3)$ groups may be differentiated using the $\mathrm{miR}-125 \mathrm{a} / E R B B 2$ ratio.

The RT-PCR kit supplier recommended using RNU6B as a reference and fold-change relative to a calibrator sample $\left(2^{\Delta \Delta C T}\right)$ for relative expression analysis (31). The present study was verified by analysing each of the 6 miRNAs and using RNU6B as reference, according to the manufacturer's instructions, thereby enabling the comparison of the present results with the previously published results regarding miRNA levels in breast cancer cells $(2,7,17-19,30,37-42)$. This analysis, using RNU6B as a reference, revealed a significantly higher expression of miR-17 in basal compared with luminal samples (MW; $\mathrm{P}=0.0366$ ). A higher level of miR-17 expression was observed in ER- and PR-negative tissues, compared with ER- and PR-positive samples. Dvigne et al (2) demonstrated that $17-5 \mathrm{p}$ and $17-3 \mathrm{p}^{*}$ exhibit highly similar expression patterns. The present results were similar to those of previous studies, in which miR-17-3p was increased in basal compared with luminal breast cancer tissue $(2,37)$. The authors also demonstrated that miR-17-5p and $3 p$ were increased in ER-negative and high-grade breast cancer tissues (2). There are contradictory data concerning miR-17-3p, which indicates that it is decreased in primary breast cancer (ductal carcinoma in situ) compared with normal epithelial tissues (38). However, these data are not directly contradictory to the present results, as the current study did not compare normal tissues with cancerous tissues; rather it compared different types of cancer.

Let-7a expression measured relative to RNU6B was lower in higher-grade samples than that in lower-grade samples. It was also lower in ER-negative samples, lymph node-positive samples and basal samples, compared with luminal samples. This finding was similar to that obtained in earlier studies. In those studies, the expression of let-7a was lower in breast cancer compared with normal tissue, and was lower in invasive ductal carcinoma with lymph node metastasis compared with those without lymph node metastasis $(7,19)$.

In the present study, a lower expression of miR-27b was observed in higher-grade cancer samples. Depending on 
the method used, the data concerning the expression of pre-miR-27b in breast cancer have been inconsistent in previous studies, and have been reported as being either lower in cancerous tissues compared with noncancerous tissues (17) or higher $(18,39)$.

Decreased expression of miR-125a was observed in higher-grade cancer and in ER-negative and luminal cancer compared with basal types of cancer. miR-125a expression has been observed to be reduced in breast cancer compared with normal tissue (19). A decreased expression of miR-125b was observed in ER- and PR-positive cancer, and in samples from larger tumours (pT1). miR-125b has been observed to be decreased in pre-invasive breast cancer compared with normal epithelium, in breast cancer that has metastasised to the lymph nodes compared with primary breast cancer, and in luminal compared with basal breast cancer $(19,38,40,41)$.

Decreased levels of miR-206 were observed in lymph node-positive cancer, in ER-positive cancer, smaller tumours, and in luminal compared with basal samples. In previous studies, miR-206 has been demonstrated to be decreased in normal breast tissues compared with primary breast tumours and in ER-positive compared with ER-negative tumours (19). In recent studies, miR-206 expression was found to be reduced in breast tumours and was inversely correlated with tumour aggressiveness $(30,42)$.

Various miRNA biomarkers have been proposed, with which to classify breast cancer cells and to predict their invasiveness and metastatic potential $(43,44)$. However, these markers are not comparable with those used in the present study, due to differences in the classification criteria used. It was confirmed that the selected miRNAs exhibited concomitant expression, as performed in array studies $(2,19)$. The aim of the present study was to improve the classification power of miRNA biomarkers, particularly in a small group of samples. Therefore, paired miRNA biomarker selection was applied as an alternative analysis method for the present data.

In order to determine potential reference miRNAs, and the most stably expressed miRNAs, samples were analysed using the GeNorme software. miR-125a, miR-125b and RNU6B were the most stable in the present group of samples. Their average expression stability was: miR-125a, 0.115; miR-125b, 0.119; RNU6B, 0.125; and let-7a, 0.206. The present analysis includes let-7a, which has been proposed for normalisation in breast cancer studies (45). Due to contradictory data regarding which miRNAs are the best reference miRNAs in breast cancer analyses, a method was proposed for selecting pairs of miRNA markers without defining the target or reference genes. In the 8 pairs of selected markers used in the present study, the most stably expressed miRNAs selected by GeNorme were miR-125b and miR-125a, which were applied to six pairs as either divisor or dividend.

Normalisation is important for the measurement of gene expression. Several normalisation approaches are used in miRNA investigations, depending on the type of platform, including single RT-qPCR assays, RT-qPCR arrays, microarrays or sequencing $(46,47)$. The most common method for normalisation in RT-qPCR assays is the application of the least variable miRNA or other small RNA, such as RNU6B RNA, as a reference (45). A relative analysis method was selected for the miRNA levels, based on the selection of one of all possible
miRNA pairs. This approach is termed 'self-normalising biomarker' or 'DiffPairs', which indicates the most effective means by which to differentiate two groups of samples $(32,48)$. The method has been applied in miRNA investigations in the brain, pancreatic cyst and lung cancer analysis, but has not, to the best of our knowledge, been assessed in breast cancer cells $(32,48,49)$. In the present study, a modified method of (diffpairs), also termed 'self-normalising biomarker', was applied to evaluate the best pair of miRNAs (32). The method was modified with regard to the anti-correlation of two miRNAs by introducing a mathematical formula that achieves lower P-values, which may aid differentiation of the groups in which at least one pair of miRNAs was inversely correlated (32).

A limitation of the present study was the small number of samples. However, the aim of the study was to provide an example of how to identify pairs of self-normalising biomarkers. Further investigation is required in order to assess the use of these markers on a greater number of patients.

The present study aimed to demonstrate a calculation method with improved diagnostic power that allows the use of a small number of miRNAs as molecular markers. In addition to the modified mathematical model, potential new pairs of markers were determined for tumour grades ( $\mathrm{G}$ classification). miR-17/miR-27b discriminated grades G2 and G3, and miR-125a/RNU6B discriminated grades G1 and G3, and grades G1 and G2. For other classification parameters, the following ratios were selected: $\mathrm{miR}-125 \mathrm{~b} / \mathrm{miR}-206$ for $\mathrm{pT}$, let-7a/RNU6B for $\mathrm{pN}$, let-7a/miR-125b for ER and PR. HER2 negative and positive groups may be differentiated using the $\mathrm{miR}-125 \mathrm{a} / \mathrm{ERBB} 2$ ratio.

In conclusion, the originality of the present study was the demonstration of an approach for improving the calculation of gene expression using a method that is able to detect and recalculate the anti-correlating expression results of two genes. The method termed 'DiffPairs' was modified and adapted to this approach for the first time, to the best of our knowledge, in order to analyse miRNA expression in breast cancer cells (32). Defining a single reference gene was abandoned, and instead the optimum specificity and sensitivity marker pairs of miRNA:miRNA, miRNA:RNU6B or miRNA:mRNA were selected. The present approach may be complimentary to a subjective classification based on the results of immunohistochemical analysis.

Future studies may help to elucidate the number of miRNA paired markers, which is required to diagnose a single sample with a similar or higher accuracy than immunohistochemistry.

\section{Acknowledgements}

The present study was supported by the National Science Centre (grant no. N N 403598538). The authors would like to thank Ms. Beata Raczak and Ms. Bogumila Ratajczak for their assistance during the preparation of this manuscript.

\section{References}

1. Ebert MS and Sharp PA: Roles for microRNAs in conferring robustness to biological processes. Cell 149: 515-524, 2012.

2. Dvinge H, Git A, Graf S, et al: The shaping and functional consequences of the microRNA landscape in breast cancer. Nature 497: 378-382, 2013. 
3. Mogilyansky E and Rigoutsos I: The miR-17/92 cluster: a comprehensive update on its genomics, genetics, functions and increasingly important and numerous roles in health and disease. Cell Death Differ 20: 1603-1614, 2013.

4. Croft L, Szklarczyk D, Jensen LJ, et al: Multiple independent analyses reveal only transcription factors as an enriched functional class associated with microRNAs. BMC Syst Biol 6: 90, 2012.

5. Lehmann U: Aberrant DNA methylation of microRNA genes in human breast cancer-a critical appraisal. Cell Tissue Res 356 657-664, 2014

6. Castells A, Gusella JF, Ramesh V, et al: A region of deletion on chromosome 22q13 is common to human breast and colorectal cancers. Cancer Res 60: 2836-2839, 2000.

7. Hu X, Guo J, Zheng L, et al: The heterochronic microRNA let-7 inhibits cell motility by regulating the genes in the actin cytoskeleton pathway in breast cancer. Mol Cancer Res 11: 240-250, 2013.

8. Kim SJ, Shin JY, Lee KD, et al: MicroRNA let-7a suppresses breast cancer cell migration and invasion through downregulation of $\mathrm{C}-\mathrm{C}$ chemokine receptor type 7. Breast Cancer Res 14: R14, 2012.

9. Wang X, Cao L, Wang Y, et al: Regulation of let-7 and its target oncogenes (Review). Oncol Lett 3: 955-960, 2012.

10. Volinia S, Calin GA, Liu CG, et al: A microRNA expression signature of human solid tumors defines cancer gene targets. Proc Natl Acad Sci USA 103: 2257-2261, 2006.

11. Santos-Jr GC, Goes AC, Vitto H, et al: Genomic instability at the 13q31 locus and somatic mtDNA mutation in the D-loop site correlate with tumor aggressiveness in sporadic Brazilian breast cancer cases. Clinics (Sao Paulo) 67: 1181-1190, 2012.

12. Olive V, Li Q and He L: mir-17-92: a polycistronic oncomir with pleiotropic functions. Immunol Rev 253: 158-166, 2013.

13. Hossain A, Kuo MT and Saunders GF: Mir-17-5p regulates breast cancer cell proliferation by inhibiting translation of AIB1 mRNA. Mol Cell Biol 26: 8191-8201, 2006.

14. Yu Z, Wang C, Wang M, et al: A cyclin D1/microRNA 17/20 regulatory feedback loop in control of breast cancer cell proliferation. J Cell Biol 182: 509-517, 2008.

15. Yu Z, Willmarth NE, Zhou J, et al: microRNA 17/20 inhibits cellular invasion and tumor metastasis in breast cancer by heterotypic signaling. Proc Natl Acad Sci USA 107: 8231-8236, 2010.

16. Sinha S, Singh RK, Alam N, et al: Alterations in candidate genes PHF2, FANCC, PTCH1 and XPA at chromosomal 9q22.3 region: pathological significance in early- and late-onset breast carcinoma. Mol Cancer 7: 84, 2008.

17. Tsuchiya Y, Nakajima M, Takagi S, et al: MicroRNA regulates the expression of human cytochrome P450 1B1. Cancer Res 66 9090-9098, 2006.

18. Jin L, Wessely O, Marcusson EG, et al: Prooncogenic factors miR-23b and miR-27b are regulated by Her2/Neu, EGF and TNF-alpha in breast cancer. Cancer Res 73: 2884-2896, 2013.

19. Iorio MV, Ferracin M, Liu CG, et al: MicroRNA gene expression deregulation in human breast cancer. Cancer Res 65: 7065-7070, 2005 .

20. Gentile M, Olsen K, Dufmats M, et al: Frequent allelic losses at 11q24.1-q25 in young women with breast cancer: association with poor survival. Br J Cancer 80: 843-849, 1999.

21. Feliciano A, Castellvi J, Artero-Castro A, et al: miR-125b acts as a tumor suppressor in breast tumorigenesis via its novel direct targets ENPEP, CK2-alpha, CCNJ and MEGF9. PLoS One 8: e76247, 2013.

22. Serpico D, Molino L and Di Cosimo S: microRNAs in breast cancer development and treatment. Cancer Treat Rev 40: 595-604, 2014

23. Scott GK, Goga A, Bhaumik D, et al: Coordinate suppression of ERBB2 and ERBB3 by enforced expression of micro-RNA miR-125a or miR-125b. J Biol Chem 282: 1479-1486, 2007.

24. Zhang Y, Yan LX, Wu QN, et al: miR-125b is methylated and functions as a tumor suppressor by regulating the ETS1 proto-oncogene in human invasive breast cancer. Cancer Res 71 3552-3562, 2011

25. Nexo BA, Vogel U, Olsen A, et al: Linkage disequilibrium mapping of a breast cancer susceptibility locus near RAI/PPP1R13 L/iASPP. BMC Med Genet 9: 56, 2008.

26. Nohata N, Hanazawa T, Enokida H, et al: microRNA-1/133a and microRNA-206/133b clusters: dysregulation and functional roles in human cancers. Oncotarget 3: 9-21, 2012.
27. Adams BD, Furneaux $\mathrm{H}$ and White BA: The micro-ribonucleic acid (miRNA) miR-206 targets the human estrogen receptor-alpha (ERalpha) and represses ERalpha messenger RNA and protein expression in breast cancer cell lines. Mol Endocrinol 21: $1132-1147,2007$

28. Song G, Zhang Y and Wang L: MicroRNA-206 targets notch3, activates apoptosis and inhibits tumor cell migration and focus formation. J Biol Chem 284: 31921-31927, 2009.

29. Yan D, Dong Xda E, Chen X, et al: MicroRNA-1/206 targets c-Met and inhibits rhabdomyosarcoma development. J Biol Chem 284: 29596-29604, 2009

30. Zhou J, Tian Y, Li J, et al: miR-206 is down-regulated in breast cancer and inhibits cell proliferation through the up-regulation of cyclinD2. Biochem Biophys Res Commun 433: 207-212, 2013.

31. Applied Biosystems. Endogenous Controls for Real-Time Quantitation of miRNA Using TaqMan MicroRNA Assays. 2010.

32. Matthaei H, Wylie D, Lloyd MB, et al: miRNA biomarkers in cyst fluid augment the diagnosis and management of pancreatic cysts. Clin Cancer Res 18: 4713-4724, 2012.

33. Pfaffl MW, Tichopad A,Prgomet C, et al: Determination of stable housekeeping genes, differentially regulated target genes and sample integrity: BestKeeper-Excel-based tool using pair-wise correlations. Biotechnol Lett 26: 509-515, 2004.

34. Escobar PF, Patrick RJ, Rybicki LA, et al: The 2003 revised TNM staging system for breast cancer: Results of stage re-classification on survival and future comparisons among stage groups. Ann Surg Oncol 14: 143-147, 2007.

35. Elston CW and Ellis IO: Pathological prognostic factors in breast cancer. I. The value of histological grade in breast cancer: Experience from a large study with long-term follow-up. Histopathology 19: 403-410, 1991

36. Corcoran C, Friel AM, Duffy MJ, et al: Intracellular and extracellular microRNAs in breast cancer. Clin Chem 57: 18-32, 2011.

37. Calvano Filho CM, Calvano-Mendes DC, Carvalho KC, et al: Triple-negative and luminal A breast tumors: differential expression of miR-18a-5p, miR-17-5p and miR-20a-5p. Tumour Biol 35: 7733-7741, 2014.

38. Hannafon BN, Sebastiani P, de las Morenas A, et al: Expression of microRNA and their gene targets are dysregulated in preinvasive breast cancer. Breast Cancer Res 13: R24, 2011.

39. Wang Y, Rathinam R, Walch A, et al: ST14 (suppression of tumorigenicity 14) gene is a target for miR-27b and the inhibitory effect of ST14 on cell growth is independent of miR-27b regulation. J Biol Chem 284: 23094-23106, 2009.

40. Baffa R, Fassan M, Volinia S, et al: MicroRNA expression profiling of human metastatic cancers identifies cancer gene targets. J Pathol 219: 214-221, 2009.

41. Bockmeyer CL, Christgen M, Muller M, et al: MicroRNA profiles of healthy basal and luminal mammary epithelial cells are distinct and reflected in different breast cancer subtypes. Breast Cancer Res Treat 130: 735-745, 2011.

42. Li Y, Hong F and Yu Z: Decreased expression of microRNA-206 in breast cancer and its association with disease characteristics and patient survival. J Int Med Res 41: 596-602, 2013.

43. Falkenberg N, Anastasov N, Rappl K, et al: MiR-221/-222 differentiate prognostic groups in advanced breast cancers and influence cell invasion. Br J Cancer 109: 2714-2723, 2013.

44. Lerebours F, Cizeron-Clairac G, Susini A, et al: miRNA expression profiling of inflammatory breast cancer identifies a 5-miRNA signature predictive of breast tumor aggressiveness. Int J Cancer 133: 1614-1623, 2013.

45. Davoren PA, McNeill RE, Lowery AJ, et al: Identification of suitable endogenous control genes for microRNA gene expression analysis in human breast cancer. BMC Mol Biol 9: 76, 2008.

46. Meyer SU, Pfaffl MW and Ulbrich SE: Normalization strategies for microRNA profiling experiments: a 'normal' way to a hidden layer of complexity? Biotechnol Lett 32: 1777-1788, 2010.

47. Qureshi R and Sacan A: A novel method for the normalization of microRNA RT-PCR data. BMC Med Genomics 6 (Suppl 1): 14,2013

48. Sheinerman KS, Tsivinsky VG, Crawford F, et al: Plasma microRNA biomarkers for detection of mild cognitive impairment. Aging (Albany NY) 4: 590-605, 2012.

49. Hennessey PT, Sanford T, Choudhary A, et al: Serum microRNA biomarkers for detection of non-small cell lung cancer. PLoS One 7: e32307, 2012 